\title{
APENDICITE AGUDA: ANÁLISE INSTITUCIONAL NO MANEJO PERI- OPERATÓRIO
}

\section{Acute appendicitis: institutional evaluation in the peri-operative managment}

\author{
Orli FRANZON, Maria Claudia PICCOLI, Thaís Torres NEVES, Marília Granzotto VOLPATO
}

ABCDDV/645

Franzon O, Piccoli MC, Neves TT, Volpato MG. Apendicite aguda: análise institucional no manejo peri-operatório. ABCD Arq Bras Cir Dig 2009;22(2):72-5 RESUMO - Racional - Apendicite aguda é a causa mais comum de abdômen agudo, responsável por elevada morbidade. Uma vez que o diagnóstico correto e precoce permanece um desafio, a compreensão de seu manejo peri-operatório é fundamental. Objetivo - Descrever o perfil clínico e avaliar o manejo peri-operatório de pacientes adultos submetidos à apendicectomia laparotômica. Métodos - Foram analisados prospectivamente 88 pacientes submetidos à apendicectomia no período de nove meses. Todos os pacientes foram operados por laparotomia, e o tratamento cirúrgico instituído obedeceu ao padrão convencional de ressecção cirúrgica por incisão Davis-Rockey, McBurney ou mediana infra-umbilical. Foi realizada análise descritiva das prevalências segundo idade, sexo, sintomatologia e resultados de exames de imagem. Antibioticoterapia foi realizada com ciprofloxacin + metronidazol ou ampicilina-sulbactam. Os pacientes foram acompanhados durante o período de internação quanto à ocorrência de complicações precoces e tempo de permanência hospitalar. Resultados - Trinta pacientes foram submetidos à ultrassonografia (56,7\% mulheres) e cinco à tomografia computadorizada - todos do sexo feminino. O leucograma diferencial foi diretamente relacionado com fases avançadas de apendicite, guardando relação com aumento do número de bastões e segmentados e queda no número de eosinófilos e linfócitos. Sessenta pacientes $(67 \%)$ fizeram antibioticoterapia e $38,33 \%$ deles tiveram perfuração apendicular. Complicações ocorreram em $23,8 \%$ dos casos, sendo que $11,4 \%$ relacionadas à infecção de ferida operatória em maior proporção relacionadas às fases avançadas de apendicite. Conclusão - Quanto maior a porcentagem de formas jovens na contagem leucocitária, mais avançada está a fase do processo inflamatório apendicular. As mulheres utilizam a ultrassonografia abdominal para esclarecimento diagnóstico em maior número do que os homens. Quanto mais avançado o estágio de evolução da apendicite, maior a prevalência de complicações.

DESCRITORES - Apendicite aguda. Complicações pós-operatórias. Apendicectomia.

\section{INTRODUÇÃO}

A apendicite aguda é a causa mais comum de abdômen agudo na criança, no adolescente e no adulto jovem com um pico de incidência na $2^{\circ}$ e $3^{\circ}$ décadas de vida. $\mathrm{O}$ risco de um indivíduo apresenta-la durante a vida inteira é de $7 \%{ }^{7,25,26}$. Sua incidência reduziu-se nas últimas décadas e as razões desse declínio ainda não estão esclarecidas, tendo sido atribuídas às mudanças nos hábitos dietéticos e na flora intestinal, melhora da nutrição, maior ingestão de vitaminas e outros ${ }^{16}$.

A obstrução da luz apendicular é o principal fator iniciante do processo inflamatório, sendo as causas mais frequentes a hiperplasia do tecido linfóide principalmente nas crianças, fecalitos, corpos estranhos (sementes, fibras, bário, projétil de arma de fogo), vermes e neoplasias (do apêndice, ceco ou metastática). A teoria obstrutiva não explica todos os casos de apendicite aguda sendo alguns atribuídos à infecção bacteriana primária do apêndice , $7,25,26^{\text {. }}$

A dor abdominal é o sintoma mais importante e o mais frequente da apendicite aguda, com migração clássica de

Trabalho realizado no Hospital Regional de São José - Dr. Homero de Miranda Gomes, São José, SC, Brasil

Endereço para correspondência: Maria Claudia Piccoli, e-mail: mcpiccoli06@yahoo.com.br periumbilical ou epigástrica para localização em fossa ilíaca direita em $2 / 3$ dos pacientes. Eventualmente pode ser referida em outros locais dependendo da posição ocupada pelo apêndice cecal, ocorrendo em $75 \%$ das vezes localização intra-peritoneal retrocecal ${ }^{1,13,24,27,28}$.

É de diagnóstico eminentemente clínico e geralmente não necessita de exames complementares. No entanto, principalmente diante de um quadro clínico duvidoso, exames laboratoriais e de imagem, sobretudo ultrassonografia e tomografia computadorizada são empregados fornecendo suporte secundário ao diagnóstico ${ }^{5,29}$.

A maioria dos cirurgiões opta pelo tratamento cirúrgico imediato $^{11}$, escolha adotada pelo serviço dos autores, mas a tendência atual de tratamento inicial conservador em casos selecionados é fato bem descrito na literatura com consistentes resultados ${ }^{2,4,6,8,10,30}$. Também, o emprego da laparoscopia diagnóstica e terapêutica principalmente em pacientes obesos e do sexo feminino tem seu inquestionável valor, porém não é assunto da presente trabalho ${ }^{14,15,18}$.

O objetivo deste estudo no período peri-operatório é determinar o perfil clínico, laboratorial e de imagem dos pacientes com apendicite aguda, os achados patológicos trans-operatórios e sua relação com o leucograma diferencial e ocorrência precoce de complicações, correlacionando-se estas variáveis entre si. 


\section{MÉTODOS}

Foram analisados prospectivamente 88 pacientes adultos submetidos à apendicectomia no período de janeiro a setembro de 2008 no Serviço de Cirurgia Geral do Hospital Regional de São José - Dr. Homero Miranda Gomes, São José, SC, Brasil. O estudo incluiu os pacientes com quadro suspeito de apendicite aguda submetidos à cirurgia, com posterior confirmação anatomo-patológica. Os critérios de exclusão foram: pacientes levados à operação com diferente diagnóstico final e os sem rotina laboratorial documentada.

Todos foram operados por laparotomia, e o tratamento cirúrgico instituído obedeceu ao padrão convencional de ressecção cirúrgica. O tipo de incisão Davis-Rockey, McBurney ou mediana infra-umbilical dependeu da escolha do cirurgião de plantão. A colocação de drenos não foi avaliada por esse estudo.

Foi analisada a prevalência da apendicite aguda segundo idade, sexo, sintomatologia, resultados do leucograma e de exames de imagem. O uso e duração da antibioticoterapia realizada com ciprofloxacin + metronidazol $(500$ mg EV 6/6h e 1,5g EV/24h respectivamente) ou ampicilina-sulbactam (1,5g EV 6/6h) foi baseado nos achados trans-operatórios. A suspensão do medicamento deu-se na ausência de febre, leucocitose e taquicardia. A fase do processo inflamatório apendicular também foi avaliada e incluída em uma das seguintes condições: catarral, supurativa, gangrenosa e perfurativa.

Os pacientes foram acompanhados durante o período de internação quanto à ocorrência de complicações precoces e tempo de permanência hospitalar.

Os dados colhidos foram inseridos em um protocolo de pesquisa, que incluiu as variáveis de interesse para posterior análise estatística no SPSS 8.0 e no EpiInfo 6.0 para suas correlações.

\section{RESULTADOS}

\section{Dados pré-operatórios}

Do total de 88 pacientes submetidos à cirurgia, 58 eram homens (65.9\%). A média de idade foi de 30,9 anos (DP $13,3)$ sendo que a moda apresentada pelo estudo estatístico foi de 20 anos.

Quanto à localização inicial da dor na admissão, 12 pacientes $(13,6 \%)$ queixavam dor difusa, $24(27,3 \%)$ epigástrica; $40(45,5 \%)$ em fossa ilíaca direita; $1(1,1 \%) \mathrm{em}$ hipocôndrio direito e $11(12,5 \%)$ em hipogástrio. Sessenta e quatro $(72,7 \%)$ apresentavam náuseas e/ou vômito; 12 $(13,6 \%)$ alteração de hábito intestinal - diarréia e parada de eliminação de gases e fezes em sete $(8,0 \%)$ e cinco $(5,7 \%)$ respectivamente. Observou-se em 51 pacientes $(58 \%)$ anorexia e em $35(39,6 \%)$ febre - temperatura axilar maior ou igual a $37,8^{\circ} \mathrm{C}$ (Tabela 1$)$.

O valor médio dos leucócitos séricos foi de 16,829 mil leucócitos $/ \mathrm{mm}^{3}$. A evolução do processo inflamatório correlacionou-se diretamente com a maior prevalência de formas jovens leucocitárias (bastões $[P<0,05]$, segmen- tados $[P<0,05])$ e com menor prevalência das séries de monócitos $(P=0,098)$, linfócitos $(P<0,05)$ e eosinófilos $(P<0,0027)$.

TABELA 1 - Perfil epidemiológico da amostra estudada $(n=88)$

\begin{tabular}{ll}
\hline Variáveis & Resultados \\
\hline Idade (média em anos) & 30,93 \\
Sexo (\%) & $65,9 \%$ masculino \\
Início da dor (\%) & $45,5 \%$ fossa ilíaca direita \\
& $27,3 \%$ epigástrica \\
& $13,6 \%$ difusa \\
& $12,5 \%$ hipogástrio \\
& $1,1 \%$ hipocôndrio direito \\
& $72,7 \%$ \\
Náuseas/vômitos (\%) & $8 \%$ \\
Diarréia (\%) & $39,6 \%$ \\
Febre (\%) & $58 \%$ \\
Anorexia (\%) & 16,829 \\
Leucócitos séricos (média $\mathrm{em} \mathrm{mm}^{3}$ ) & \\
\hline
\end{tabular}

Em relação aos métodos de imagem, 30 pacientes $(34,1 \%)$ foram submetidos à ultrassonografia, e cinco $(5,7 \%)$ - todos do sexo feminino - à tomografia computadorizada de abdome para esclarecimento diagnóstico. Foi observado que o sexo feminino apresentou 4,9 vezes mais chance de realizar ultrassonografia - OR 4,90 $(1,68-14,64)$ comparado ao sexo masculino - (Tabela 2).

TABELA 2 - Uso da ultrassonografia no diagnóstico de apendicite aguda de acordo com sexo

\begin{tabular}{llll}
\hline Sexo & \multicolumn{2}{l}{ Ultrassonografia* } & Total \\
& Realizada & Não realizada & \\
\hline Feminino & $17(56,7 \%)$ & $13(43,35)$ & $30(100 \%)$ \\
Masculino & $13(22,4 \%)$ & $45(77,6 \%)$ & $58(100 \%)$ \\
\hline
\end{tabular}

$* P=0,00086$

\section{Dados trans-operatórios}

Quanto ao processo inflamatório encontrado, 15 pacientes $(17 \%)$ apresentavam-se na fase I; $31(35,2 \%)$ na II; $17(19,3 \%)$ na III e $25(28,4 \%)$ na IV.

\section{Dados pós-operatórios}

O tempo médio de internação foi de 3.7 dias (DP 3,057). A média de tempo entre a admissão na emergência a operação foi de 0,35 dias (DP 0,95$)(P<0,05)$.

Sessenta pacientes $(67 \%)$ fizeram uso de antibioticoterapia com média de duração de 4,93 dias (DP 5,06 e moda $=2$ ) e ainda, quanto maior o grau do processo inflamatório apendicular, maior foi a prevalência desta terapia (Tabela 3).

TABELA 3 - Correlação entre a fase do processo inflamatório apendicular com a antibioticoterapia

\begin{tabular}{llllll}
\hline Antibioticoterapia & \multicolumn{4}{c}{ Fases da apendicite* } & \multicolumn{1}{c}{ Total } \\
\hline & \multicolumn{1}{c}{ I } & II & III & IV & \\
Realizada & $5(8,34 \%)$ & $18(30 \%)$ & $14(23,33 \%)$ & $23(38,33 \%)$ & $60(100 \%)$ \\
Não realizada & $10(35,72 \%)$ & $13(46,42 \%)$ & $3(10,72 \%)$ & $2(7,14 \%)$ & $28(100 \%)$ \\
\hline
\end{tabular}

* $P=0,00020$ 
Dezenove pacientes $(23,8 \%)$ apresentaram complicações, sendo a mais comum infecção de ferida operatória $(11,4 \%)$ - (Figura 1).

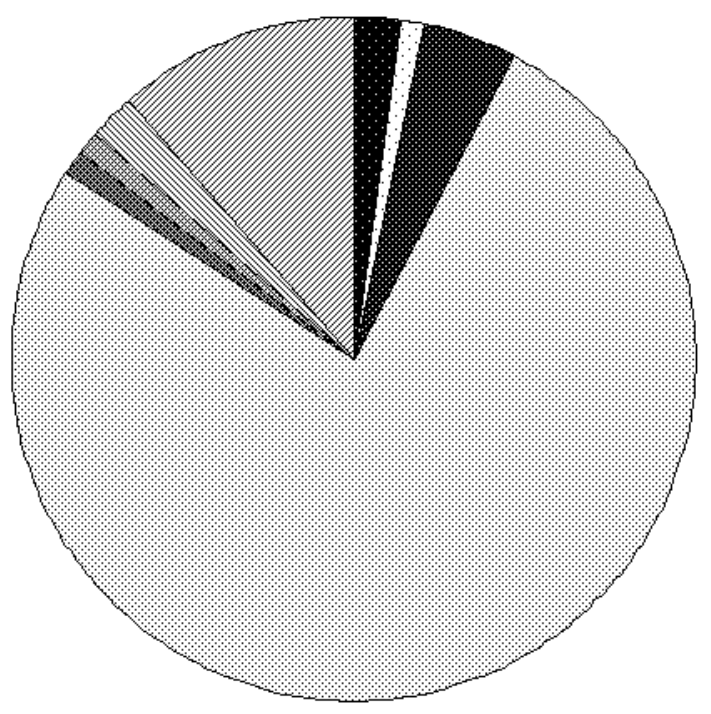

\begin{tabular}{|c|c|}
\hline - Peritonite & घltu \\
\hline abcesso intra-cavitario & هusência de complicaçốes \\
\hline 国 fístula fecal & 图 $\mathrm{HDA}$ \\
\hline $\mathbb{\$}$ hérnia interna & छInfecçẫo $\mathrm{FO}$ \\
\hline
\end{tabular}

FIGURA 1 - Prevalência de complicações pós-operatórias.

Por fim, observou-se correlação direta entre a presença de complicações precoces e a fase do processo inflamatório apendicular (Tabela 4).

TABELA 4 - Correlação entre a fase do processo inflamatório apendicular e a presença de complicações pósoperatórias

\begin{tabular}{|c|c|c|c|c|c|}
\hline Complicações & & Fases da : & pendicite* & & Total \\
\hline & I & II & III & IV & \\
\hline Sim & $2(10,5 \%)$ & $2(10,5 \%)$ & $4(21 \%)$ & $11(57,9 \%)$ & $19(100 \%)$ \\
\hline Não & $13(18,84 \%)$ & $29(42,02 \%)$ & $13(18,86 \%)$ & $14(20,28 \%)$ & $69(100 \%)$ \\
\hline
\end{tabular}

$* P=0,016$

\section{DISCUSSÃO}

A apendicite aguda apresenta maior prevalência no sexo masculino, em pacientes com média de idade de 30,93 anos (DP13, 38), com clássica apresentação inicial da dor em epigástrio ou peri-umbilical ${ }^{3,7,9,13,16,24,25,26,27,28}$. Porém, os pacientes desta amostra apresentaram predomínio do início da dor em fossa ilíaca direita $(45,5 \%)$. Náuseas e vômitos surgem na grande maioria dos casos, chegando a prevalências de até $90 \%{ }^{7,9,13,16,24,25,26,27,28}$ confirmando o valor encontrado neste de estudo de $72,7 \%$. Anorexia e febre podem acompanhar o quadro com frequência de $58 \%$ e $39,6 \%$ respectivamente ${ }^{7,25,26}$.

Nos exames laboratoriais, o hemograma mostra leucocitose entre 12000 e $18000^{3}$. Ainda, se a contagem diferencial mostrar desvio significativo para as formas imaturas e houver redução ou desaparecimento das séries de monócitos, eosinófilos, e/ou linfócitos, a probabilidade de apendicite com perfuração é maior ${ }^{3,17,23}$. O presente estudo obteve valores estatisticamente significativos para as séries de bastões e segmentados assim como para os eosinófilos e linfócitos.

A ultrassonografia abdominal foi o exame de imagem mais empregado no caso de dúvida diagnóstica, em função da facilidade de realização, baixo custo e acurácia ${ }^{5,29}$. Trinta pacientes foram submetidos a esse exame, sendo $56,7 \%$ do sexo feminino. A tomografia computadorizada, reservada para dúvida diagnóstica foi realizada em apenas 5,7\% dos pacientes, todos do sexo feminino.

O tempo médio entre a admissão na emergência e a sala cirúrgica foi relativamente curto, de 0,35 dias (DP 0,95), com $96,6 \%$ dos pacientes operados em até $24 \mathrm{hs}$ da internação, evidenciando-se diagnóstico e tratamento precoces.

A antibioticoterapia foi empregada em $67 \%$ dos pacientes, com média de 4,93 dias (DP5,06) sendo mais utilizada em fases avançadas do processo inflamatório. Apesar disso, este estudo observou altas prevalências de seu uso em fases precoces $-8,34 \%$ e $30 \%$ respectivamente para as fases $1 \mathrm{e}$ 2 - mesmo com consenso geral de que esquema terapêutico deva ser utilizado por no máximo $24 \mathrm{~h}$ nos casos não complicados $^{16,23}$. Talvez esses valores sejam reflexo do maior número de pacientes em fase 2 da amostra analisada, ou, por insegurança do cirurgião na ausência de terapia antimicrobiana para seu paciente, mesmo em fases precoces.

Apesar da mortalidade da apendicite aguda ser atualmente baixa (inferior a $0,1 \%$ nos casos não complicados e 3-5\% nas perfuradas), a sua morbidade permanece ainda elevada, com incidência geral de complicações de $10 \% \%^{12,19,20,21,22,31}$ predominantemente em fases mais avançadas.

O presente estudo não apresentou óbitos e incidência de complicações ligeiramente acima daquela descrita na literatura $^{12,19,21}$ de 23,8\%, respondendo pela metade dos casos a infecção de parede abdominal. Outras complicações pós-operatórias precoces menos frequentes encontradas neste estudo, porém mais graves, foram obstrução intestinal por hérnia interna $(2,3 \%)$, abscesso intra-cavitário $(4,5 \%)$ e fístula fecal $(1,1 \%)$.

Algumas limitações podem ser observadas neste estudo prospectivo. A avaliação inicial na admissão do paciente, bem como o relato dos achados trans-operatórios foram feitos mediante preenchimento de protocolo, estando sujeitosa diferentes examinadores e diferentes descrições cirúrgicas.

Também, ausência de seguimento a médio e longo prazo impossibilita avaliação precisa das complicações tardias relativas ao procedimento cirúrgico.

\section{CONCLUSÃO}

Quanto maior a porcentagem de formas jovens na contagem leucocitária, mais avançada está a fase do processo inflamatório apendicular. As mulheres utilizam a ultrassonografia abdominal para esclarecimento diagnóstico em maior número do que os homens. Quanto mais avançado o estágio de evolução da apendicite, maior a prevalência de complicações. 
Franzon O, Piccoli MC, Neves TT, Volpato MG. Acute appendicitis: institutional evaluation in the peri-operative managment. ABCD Arq Bras Cir Dig 2009;22(2):72-5

ABSTRACT - Background - Acute appendicitis is one of the most common cause of acute abdomen and is responsible for high morbidity. Correct diagnosis remains a challenge, thus accurate perioperative assessment is important in planning surgical therapy. Aim - To evaluate institutional findings in perioperative workup, operative approach and adverse outcomes in patients who underwent open surgical intervention for acute appendicitis. Method - A prospective chart was performed of 88 adults patients undergoing open appendectomy. Variables compared were imaging methods and laboratory evaluation, pathologic findings and early complications. Statistical analysis was performed by SPSS 8.0 and EpiInfo6.0. Results - Thirty patients underwent ultrassonography (56,7\% females) and five computorized tomography (all women). The differencial white cell count was directly related to more advanced phases regarding increased of "stabs", segmented and eosinophyls/lymphocytes decrease $(P>0,005)$. Sixty $(67 \%)$ patients used antibiotic therapy and $38,33 \%$ of them had perforated appendicitis. Was found $23,8 \%$ of complications, $11,4 \%$ was wound infections and patients with perforated appendicitis. Conclusion - Females demand more imaging methods. The differencial white cell count in complicated appendicitis has an increment in less mature neutrophils and reduction of the eosinophils and lymphocytes. Advanced phases has increase risk of early complications mainly superficial wound infections and shows more prevalence in using.

HEADINGS - Acute appendicitis. Surgical outcomes. Appendectomy.

\section{REFERÊNCIAS}

1. Andersson RE, Petzold MG. Nonsurgical treatment of appendiceal abscess or phlegmon: a systematic review and meta-analysis. Ann Surg 2007; 246(5):741-8.

2. Andersson RE. Meta-analysis of the clinical and laboratory diagnosis of appendicitis. Br J Surg 2004; 91(1):28-37.

3. Borges PSGN, Lima MC, Neto GHF. Validação do escore de Alvarado no diagnóstico de apendicite aguda em crianças e adolescentes no Instituto Materno Infantil de Pernambuco - IMIP. Rev Bras Saúde Matern Infant 2003; 3(4):439-445.

4. Cappendijk V, Hazebroek F. The impact of diagnostic delay on the course of acute appendicitis. Arch Dis Child 2000; 83(1):64-66.

5. Chiang DT, Tan ET, Birks D. 'To have ... or not to have'. Should Computed Tomography and Ultrasonography be Implemented as a Routine Work-Up for Patients with Suspected Acute Appendicitis in a Regional Hospital? Ann R Coll Surg Engl 2008; 90(1):17-21.

6. Clyde C, Bax T, Merg A, MacFarlane M, Lin P, Beyersdorf S, McNevin M. Timing of intervention does not affect outcome in acute appendicitis in a large community practice. The Am J of Surg 2008; 195(5):590-593.

7. Coelho JCU.Aparelho Digestivo: clínica e cirurgia. São Paulo: Editora Atheneu, 2005; V2:862-868.

8. Ditillo MF, Dziura JD, Reuven R. Is it safe to delay appendectomy in adults with acute appendicitis?Ann of Surg 2006; 244(5):656-60.

9. Druszcz CC, Borsato EP, Pinto JSP, Malafaia O. Aplicação multicêntrica informatizada da coleta de dados clínicos na apendicite aguda. ABCD Arq Bras Cir Dig 2007;20(3):175-81.

10. Eldar S, Nash E, Sabo E, Matter I, Kunin J, Mogilner JG, Abrahamson J. Delay of Surgery in Acute Appendicitis. The Am J of Surgery 1997; 173(3):194-198.

11. Faiz O, Aylin P, Bottle A. Changing trends in surgery for acute appendicitis. Br J Surg 2008; 95:363-368.

12. Gupta R, Sample C, Bamehriz F, Birch DW. Infectious complications following laparoscopic appendectomy. Can J Surg 2006; 49(6):397-400.

13. Humes DJ. Clinical review - Acute appendicitis. BMJ 2006; 333:530-534.

14. Hussain A, Mahmood H, Nicholls J, El-Hasani S. Prevention of intra-abdominal abscess following laparoscopic appendicectomy for perforated appendicitis: A prospective study. International J of Surg 2008; 6(5):374-377.

15. Júnior BS, Guimarães CA. Práticas cirúrgicas baseadas em evidências: apendicectomia laparoscópica versus a céu aberto. Rev. Col. Bras. Cir. 2008; 35(1):56-60.

16. Kang J, Hoare J, Majeed A, Williamson RCN, Maxwell JD. Decline in admission rates for acute appendicitis in England. Br J Surg 2008; 95(7):1586-1592.

17. Kasatpibal N, Norgaard M, Sorensen HT, Schonheyder HC, Jamulitrat S, Chongsuvivatwong V. Risk of surgical site infection and efficacy of antibiotic prophylaxis: a cohort study of appendectomy patients in Thailand. BMC Infect Dis. 2006; 6:111.
18. Katkhouda N, Mason RJ, Towfigh S, Gevorgyan A, Essani R.Laparoscopic Versus Open Appendectomy: A Prospective Randomized Double-Blind Study. Ann Surg 2005; 242(3):439-450

19. Kato Y, Takashi M, Satoko I, Lane GJ, Okazaki T, Yamataka A.Lapprotector ${ }^{\mathrm{TM}}$ Use Decreases Incisional Wound Infections in Cases of Perforated Appendicitis: A Prospective Study. Japan Asian J of Surg 2008; 31(3):101-103.

20. Kieran JA, Curet MJ, Ascermer CR. Institutional variations in the management of patients with acute appendicitis. J. Gastroint Surg 2003; 7(4):523-8

21. Krisher SL, Browne A, Dibbins A, Tkacz N, Curci M. Intra-abdominal Abscess After Laparoscopic Appendectomy for Perforated Appendicitis. Arch Surg $2001 ; 136: 438-441$

22. Margenthaler JA, Longo WE, Virgo KS, Johnson FE, Oprian CA, Henderson WG, Daley J, Khuri SF. Risk factors for adverse outcomes after the surgical treatment of appendicitis in adults. Ann Surg 2003; 238(1):59-66.

23. Ong CP, Chan TK, Chui CH, Jacobsen AS. Antibiotics and postoperative abscesses in complicated appendicitis: is there any association? Singapore Med J 2008; 49(8):615-8.

24. Paulson EK, Kalady M F, Pappas TN. Suspected Appendicitis. N Engl J Med 2003; 349(3):305

25. Rodrigues JJG, Machado MCC, Rasslan S. Clínica Cirúrgica. Barueri, São Paulo: Manole 2008; V2:1557-1558.

26. Saunders Company. Sabiston Textbook of Surgery: The Biological Basis of Modern Surgical Practice. Translation of the sixteenth English language edition, 2003. V2: 1008-1017.

27. Simpson J, Scholefield JH. Acute Appendicitis. The Foundation Years 2006; 2(2):72-75.

28. Singhal V, Jadhav V. Acute Appendicitis: Are we Over Diagnosing it? Ann R Coll Surg Engl 2007; 89(8):766-769.

29. Summa M, Perrone F, Priora F, Testa S, Quarati R, Spinoglio G. Integrated clinical-ultrasonographic diagnosis in acute appendicitis. J of Ultrasound 2007; 10(4):175-178.

30. Yardeni D, Hirschl RB, Drongowski RA, Teitelbaum DH, Geiger JD, Coran AG. Delayed versus immediate surgery in acute appendicitis: do we need to operate during the night? J Pediatr Surg 2004; 39(3):464-9; discussion 464-9.

31. Young YR, Chiu TF, Chen JC, Tung MS, Chang MW, Chen JH, Sheu BF. Acute Appendicitis in the Octogenarians and Beyond: A Comparison With Younger Geriatric Patients. The Am J Med Sciences 2007; 334:255-259.

Fonte de financiamento: não há Conflito de interesse: não há Recebido para publicação: 17/01/2009 Aceito para publicação: 03/03/2009 